\title{
Cell surface heat shock protein 90 modulates prostate cancer cell adhesion and invasion through the integrin-ß1/ focal adhesion kinase/c-Src signaling pathway
}

\author{
XUEGUANG LIU ${ }^{1}$, ZUOQIN YAN ${ }^{2}$, LIANG HUANG $^{3}$, MUYI GUO $^{1}$, ZHIGANG ZHANG $^{1}$ and CHANGAN GUO ${ }^{2}$ \\ ${ }^{1}$ Department of Pathology, Shanghai Medical College; ${ }^{2}$ Department of Orthopedics, Zhongshan Hospital; \\ ${ }^{3}$ Clinical Eight-Academic Year Specialty, Shanghai Medical College, Fudan University, Shanghai 200032, P.R. China
}

Received November 18, 2010; Accepted January 14, 2011

DOI: $10.3892 /$ or.2011.1202

\begin{abstract}
Heat shock protein (Hsp) 90 is a molecular chaperone that maintains the active conformation and function of numerous client oncoproteins in cancer cells. Hsp90 has also been detected on the plasma membrane of cells, and its expression has been suggested to correlate with metastatic potential. We studied the PC3 cell line, which is a highly invasive human prostate cancer cell line, and confirmed that Hsp90 is present on the cell surface of PC3 cells. Interestingly, cell surface Hsp90 was also specifically localized at the leading edge of migrating cells. By using a specific antibody that inhibited cell surface Hsp90, adhesion and invasion of PC3 cells were significantly suppressed in vitro. Concomitantly with these findings, we demonstrated that the inhibition of cell surface Hsp90 not only inhibited the FN-dependent association between FAK, c-Src and integrin $\beta 1$, but also significantly inhibited the phosphorylation of FAK and c-Src, as well as their downstream targets paxillin and p130Cas. Additionally, the Hsp90 antibody reversed cell invasion stimulated by overexpression of FAK. These data indicate that cell surface Hsp90 is involved in prostate cancer cell invasion through the integrin $\beta 1 / F A K / c-S r c$ signaling pathway. Our study provides new insights into the mechanisms of cell surface Hsp90 in cancer invasion. These results suggest that molecular targeting of cell surface Hsp90 may therefore be a novel target for the effective treatment of metastatic prostate cancer.
\end{abstract}

\section{Introduction}

The second leading cause of cancer-related death among men in industrialized countries is androgen-independent metastatic prostate carcinoma (1). Although prostate cancer is hormon-

Correspondence to: Dr Changan Guo, Department of Orthopedics, Zhongshan Hospital, Fudan University, Shanghai 200032, P.R. China E-mail: changanguo@hotmail.com

Key words: heat shock protein 90, cell surface, in vitro invasion, prostate cancer ally regulated, androgen deprivation therapy inevitably results in the progression to castrate-resistant disease, uncontrolled growth, and metastasis. Cancer invasion occurs via a combination of complex events that initially lead to changes in cell adhesive properties, allowing transformed cells to migrate, gain access to the circulation, and form metastatic colonies. Since cell motility is a critical requirement for tumor cells to acquire invasive properties, the molecules that regulate cell motility provide an attractive therapeutic target for the treatment of advanced and aggressive prostate cancer (2).

Heat shock protein 90 (Hsp90) is an essential molecular chaperone that forms a large multi-chaperone complex that mediates the proper folding, activation, and assembly of its numerous substrate, or 'client', proteins $(3,4)$. When Hsp90 is pharmacologically inhibited by Hsp90 inhibitors, such as geldanamycin (GA) and its derivatives, its client proteins become misfolded and subsequently ubiquitinated and degraded via the proteasomal pathway (3). In many cases, Hsp90 client proteins are mutated or activated in cancer cells $(3,4)$. Since many cancer cells depend heavily on these signaling proteins, the pharmacological inhibition of Hsp90 simultaneously inhibits multiple oncogenic signaling pathways and can therefore achieve a powerful anti-cancer effect (3-5). Recently, several studies have shown Hsp90 to be localized to the cell surface of certain types of cancer cells, such as melanoma (6-8), fibrosarcoma (9), breast cancer (10), bladder cancer (6), neuroblastoma (11), and neuronal cells (12). The inhibition of cell surface Hsp90 with anti-Hsp90 antibodies $(6,7,10,12)$, GA coupled to cell-impermeable agarose beads (9), or a small molecule cell-impermeable Hsp90 antagonists (6) has been reported to reduce cell migration in vitro and have a dramatic impact on the colonization of the lung by melanoma cells in vivo (6-8), suggesting that the inhibition of cell surface Hsp90 may provide a new therapeutic approach to targeting the metastatic phenotype. However, our understanding of the extracellular functions of cell surface Hsp90 is still in its early stages. Some studies have suggested that the inhibition of cell surface Hsp90 affects cytoskeletal assembly $(6,10,12)$. In addition, Tsutsumi et al found that targeting cell surface Hsp90 with a small molecule cell-impermeable Hsp90 inhibitor disrupted the fibronectin-stimulated interaction between $\mathrm{c}-\mathrm{Src}$ and integrin $\beta 1$ in bladder cancer cells (6). 
Cell surface Hsp90 may possibly modulate integrin $\beta 1 / \mathrm{c}-\mathrm{Src}$ signaling.

Cell migration is a highly coordinated process that involves the continuous formation and disassembly of focal adhesions. Integrins, which are heterodimeric transmembrane glycoproteins that are composed of non-covalently linked $\alpha$ - and $\beta$-subunits, link the extracellular matrix (ECM) to a cell's actin cytoskeleton at points of focal adhesions (13). Upon binding to ECM ligands, the integrins cluster, facilitating the autophosphorylation of FAK at tyrosine 397, which not only increases the catalytic activity of FAK but also creates a highaffinity binding site for Src family tyrosine kinases $(13,14)$. In prostate cancer cells, the FAK/Src signaling pathway and some of its downstream targets (including p130Cas and paxillin) have been proposed to be involved in regulating integrins-mediated cell migration and invasion (13-20). High levels of FAK/Src expression and signaling have been shown to be positively correlated with the invasive and migratory capacity of prostate cancer cells $(13-18,20)$. Therefore, it has been proposed that cell surface Hsp90 likely modulates PC3 cell motility by mediating integrin-FAK/Src signaling.

Although one report mentioned that PC3 cells treated with a small molecule cell-impermeable Hsp90 antagonist showed decreased motility (6), there has thus far been no report examining the expression of cell surface Hsp90 in prostate cancer cells. In this study, we demonstrated that Hsp90 is localized to the surface of metastatic human prostate cancer PC3 cells. Additionally, we found that the inhibition of cell surface Hsp90 using a cell-impermeable antibody suppressed the motility of PC3 cells as well as their capacity to participate in integrinmediated adhesion through an integrin- $\beta 1 / \mathrm{FAK} / \mathrm{c}-\mathrm{Src}$ pathway.

\section{Materials and methods}

Reagents. Anti-Hsp90 (SPA830, SPA835) antibodies were from Stressgen (MI, USA). Anti-FAK, anti-phosphorylated FAK (Y379, Y576, Y577 and Y925) antibodies, Alexa Fluor 488 or 546-conjugated secondary antibodies and streptavidin agarose beads were from Invitrogen (CA, USA). Anti-Src, anti-phosphorylated Src (Y416), anti-p130Cas, anti-phosphorylated p130Cas (Y410), anti-paxillin, and anti-phosphorylated paxillin (Y118) were obtained from Cell Signaling Technologies (MA, USA). TS2/16 monoclonal antibody, which activates integrin $\beta 1$-dependent binding to extracellular matrix substrates, was obtained from Thermo Scientific (IL, USA). Anti-integrin $\beta 1$ (HUTS-4) antibody was from Millipore (MA, USA). Anti-integrin $\beta 1$ (clone 18) antibody for immunoprecipitation was from BD Bioscience (CA USA). Anti-phosphotyrosine antibody (clone 4G10) were from Upstate Biotechnology (MA, USA). Anti-glyceraldehyde-3phosphate dehydrogenase (GAPDH, 1D4) antibody was from Santa Cruz biotechnology (CA, USA). The Src inhibitor, PP2 was from Calbiochem (NJ, USA). Sulfo-NHS-LC-Biotin, DyLight 549 Ab Labeling Kit and Zeba Micro Desalt Spin Columns were from Pierce (IL, USA). The FAK SMARTpool siRNA, control siRNA, and the transfection reagent were obtained from Dharmacon (CO, USA).

Cell culture. The human prostate cancer cell line PC3 was obtained from the American Tissue Culture Collection
(Rockville, MD, USA) and were maintained in DMEM supplemented with $10 \%$ fetal bovine serum and $1 \%$ penicillin/streptomycin in a $5 \% \mathrm{CO}_{2}$-containing humidified atmosphere.

Immunofluorescence staining. PC3 cells were cultured on coverslips coated with $10 \mu \mathrm{g} / \mathrm{ml}$ fibronectin (FN) and were incubated with an anti-Hsp90 antibody SPA830 (diluted 1:200 in culture medium) or control IgG on ice for $1 \mathrm{~h}$. They were then fixed with $2 \%$ paraformaldehyde for $10 \mathrm{~min}$ and blocked with $3 \%$ BSA-PBS for $30 \mathrm{~min}$. Cells were incubated with Alexa Fluor 546-conjugated secondary antibodies for $1 \mathrm{~h}$ at room temperature. As a positive control, cells were permeabilized with $0.1 \%$ Triton X-100 after fixation and then incubated with SPA830 to show total Hsp90 staining. Confocal images were acquired with a Leica TCS SP2 microscope.

In addition, a wound-healing assay was used to examine changes in cell surface Hsp90 expression. Briefly, PC3 cells were cultured on coverslips coated with $10 \mu \mathrm{g} / \mathrm{ml} \mathrm{FN}$. When cells reached confluence, wounds were created by scraping the coverslips with a pipette tip. After an additional 6-h incubation, immunofluorescence staining for cell surface Hsp90 was performed as described previously.

Biotinylation of cell surface proteins. Subconfluent PC3 cells were rinsed three times with cold Dulbecco's PBS (DPBS). Cell surface proteins were labeled with membrane impermeable Sulfo-NHS-LC-Biotin dissolved in DPBS at a concentration of $0.5 \mathrm{mg} / 1$ and incubated on ice for $30 \mathrm{~min}$. The labeling reaction was quenched by rinsing three times with $100-\mathrm{mm}$ glycine dissolved in DPBS. The cells were then washed once more with DPBS. Cells were then lysed using lysing buffer $(0.5 \%$ SDS, $1 \%$ Nonidet P-40, and $0.25 \%$ sodium deoxycholate) supplemented with Complete Mini protease inhibitors (Roche Diagnostics, Mannheim, Germany) for $30 \mathrm{~min}$. Biotinylated proteins were precipitated from the cell lysate using streptavidin agarose beads, separated by $10 \%$ SDS-PAGE, and analyzed using the anti-Hsp90 antibody SPA835.

Antibody internalization. Although Tsutsumi et al previously used the anti-Hsp90 antibody SPA830 to target cell surface Hsp90 via a brief incubation (6), there is no definitive data regarding whether or not it enters cells during incubation. By labeling SPA830 using the DyLight 549 Ab Labeling Kit, we examined whether this antibody was internalized. Briefly, the antibody buffer was replaced with $50-\mathrm{mM}$ sodium borate (pH 8.5) with Zeba Micro Desalt spin columns. The antibody was then incubated with DyLight Reagent for $60 \mathrm{~min}$ at room temperature protected from light. Next, the labeling reaction went through the purification resin by centrifugation. The labeled protein was protected from light and stored at $4^{\circ} \mathrm{C}$ for up to two weeks. The same amount of control IgG or BSA was also labeled. PC3 cells were treated with the labeled SPA830 for 4 and $8 \mathrm{~h}$, respectively, and were subsequently observed under the confocal microscope.

Cell adhesion assays. Adhesion assays were performed using a modified method (21). Briefly, 96-well plates were coated 
A

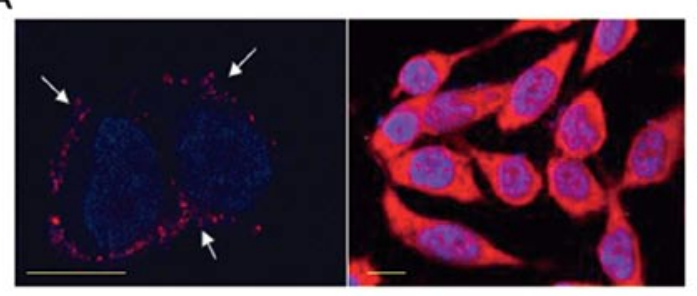

non-permeablized

permeablized

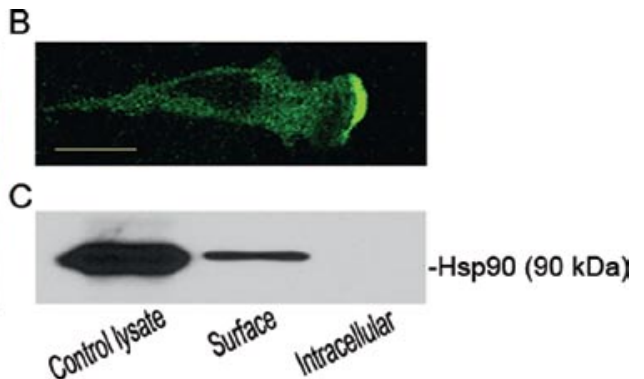

Figure 1. Validation of the localization of Hsp90 on the surface of PC3 cells. (A) PC3 cells were incubated with anti-Hsp90 antibody (SPA830) and were subsequently fixed and stained with a fluorescent secondary antibody. Total Hsp90 staining on the permeabilized cells was used as the positive control. (B) Migrating cells in a wound-healing assay were stained for cell surface Hsp90 as described above. (C) Cell surface proteins were biotinylated, immunoprecipitated (IP) by binding to streptavidin agarose beads, and then probed with anti-Hsp90 antibody (SPA835). Bar, 20.0 $\mu \mathrm{m}$.

with serial dilutions (from 0.1 to $10 \mu \mathrm{g} / \mathrm{ml}$ ) of various ECM proteins or $10 \mu \mathrm{g} / \mathrm{ml}$ poly-L-lysine in PBS. Non-specific binding sites were blocked with $0.1 \%$ BSA in PBS for $30 \mathrm{~min}$ at room temperature. PC3 cells were pretreated with SPA830 or control IgG alone in DMEM supplemented with $10 \%$ FBS (v/v) for $24 \mathrm{~h}$. Next, $2 \times 10^{5}$ cells were seeded in quintuplicate onto the coated 96 -well multiplates that were prepared as described above. Cells were allowed to adhere for $30 \mathrm{~min}$ at $37^{\circ} \mathrm{C}$. At the end of incubation period, the wells were rinsed three times with PBS to remove non-adherent cells. Cells were fixed with $3.7 \%$ paraformaldehyde for $10 \mathrm{~min}$ and stained using crystal violet. The crystal violet was then dissolved in $100 \mathrm{ml}$ of $0.1 \mathrm{~N}$ sodium citrate in $50 \%$ ethanol (v/v) and optical density was measured at $550 \mathrm{~nm}$ in an enzyme-linked immunosorbent assay (ELISA) plate reader.

In vitro invasion assay. Modified Boyden chambers (8- $\mu \mathrm{m}$ pore size, Transwell, Corning Costar, Corning, NY) were used in the cell migration assays. PC 3 cells $\left(2 \times 10^{3}\right)$ were plated into the upper chamber in $0.1 \%$ fetal bovine serum in DMEM. DMEM containing 10\% bovine serum was added to the lower compartment. Cells were pretreated for $8 \mathrm{~h}$ with the indicated reagents before they were plated into the chambers. These same reagents were added to the chambers following cell plating. The cells were allowed to invade for $24 \mathrm{~h}$ at $37^{\circ} \mathrm{C}$ in a $5 \% \mathrm{CO}_{2}$-containing humidified incubator. After incubation, the non-motile cells were removed with cotton swabs and the adherent cells that were attached to the bottom surface of the filter (the invading cells) were fixed in $3.7 \%$ formaldehyde for $10 \mathrm{~min}$ and stained with hematoxylin and eosin. The invasiveness was determined by determining the number of penetrating cells under a microscope at x400 magnification. The mean number of cells per field was recorded for ten random fields. Each experiment was performed in triplicate.

Immunoprecipitation and immunoblotting assays. After the indicated treatments, PC3 cells were detached and replated onto FN-coated wells (10 $\mu \mathrm{g} / \mathrm{ml} \mathrm{FN;} \mathrm{40,000} \mathrm{cells} \mathrm{per} \mathrm{well).}$ After incubation for $1 \mathrm{~h}$ at $37^{\circ} \mathrm{C}$, cells were lysed in ice cold lysis buffer [20 mM HEPES (pH 7.5), $100 \mathrm{mM} \mathrm{NaCl,} 1 \mathrm{mM}$ $\mathrm{MgCl}_{2}, 20 \mathrm{mM}$ sodium molybdate, $0.1 \%$ NP40, 2 mM EDTA, $10 \%$ glycerol, $5 \mathrm{mM}$ sodium fluoride, and $1 \mathrm{mM}$ sodium orthovanadate] containing a cocktail of protease inhibitors (Roche Diagnostics). Unless otherwise indicated, for all experiments, immunoprecipitations were performed overnight and protein $\mathrm{G}$ beads (Invitrogen) were washed with the respective lysis buffer and boiled in Laemmli buffer. Lysates were subjected to SDS-PAGE, transferred to a nitrocellulose membrane, and immunoblotted with antibodies against FAK pY397, FAK pY576, FAK pY577, FAK pY925, FAK, Src pY416, c-Src, p130Cas pY410, p130Cas, paxillin, paxillin pY118 (dilution 1:1000), activated integrin $\beta 1$ (HUTS-4, dilution 1:500), integrin- $\beta 1$ (clone 18, dilution 1:250, BD Biosciences, San Jose, CA ), anti-phosphotyrosine antibody (clone 4G10, dilution 1:800) or GAPDH, dilution (1:1000). Films were scanned and the optical density of the signals was measured with Kodak Digital Science ID (Eastman Kodak, USA).

Plasmids and transient transfection. PC3 cells were transfected with FAK siRNA or control non-specific siRNA according to the manufacturer's instructions. The FAK-specific siRNA sequences were: D-05 FAK siRNA, 5'-GAAGUUGGGUUGUCUAGAAUU-3'; and D-07 FAK SiRNA, 5'-GGAAAUUGCUUUGAAGUUGUU-3'. FAK-GFP Plasmid coding wild-type FAK was a gift from Dr J.T. Parsons (Department of Microbiology, University of Virginia, Charlottesville, VA, USA). Transient transfection of FAK plasmid was accomplished using Lipo2000 and Lipofectamine Plus (Invitrogen) according to the manufacturer's instruction. Cells were incubated for $36 \mathrm{~h}$ after transfection and then used for experiments.

Statistical analysis. The data represent the mean \pm standard errors from three independent experiments except where indicated. Statistical analysis was performed by Student's $\mathrm{t}$-test at a significance level of $\mathrm{P}<0.05$.

\section{Results}

Expression of Hsp90 on the surface of PC3 cells. Because there has not yet been a definitive report regarding the localization of cell surface Hsp90 on prostate cancer cells, we investigated its expression on PC3 cells at both the morphological and biochemical levels. Live PC3 cells were incubated with anti-Hsp90 antibody. The cells were subsequently fixed and labeled with the fluorescent secondary antibody. Using this fixation method without utilizing a 


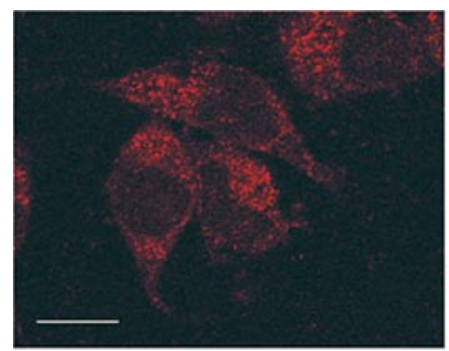

SPA830

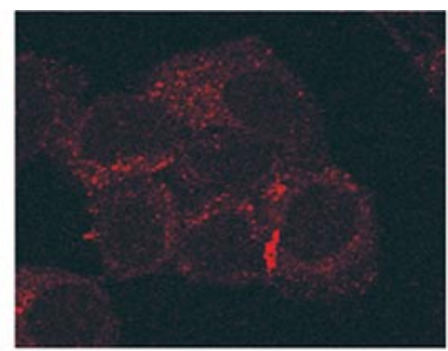

IgG control

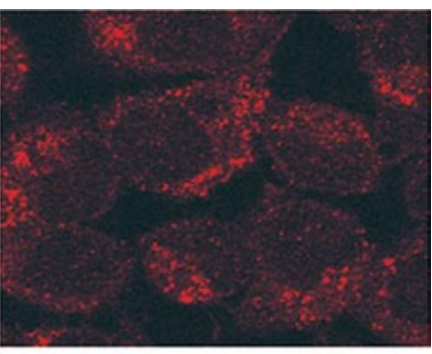

BSA

Figure 2. Hsp90 antibody SPA830 is not internalized after an 8-h incubation with PC3 cells. SPA830, control IgG, or BSA were labeled with DyLight 549 fluorochrome and were then incubated with PC3 cells. Bar, $20 \mu \mathrm{m}$.

A

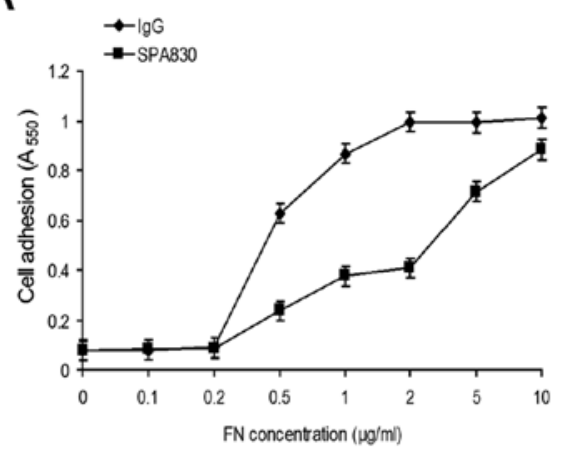

C

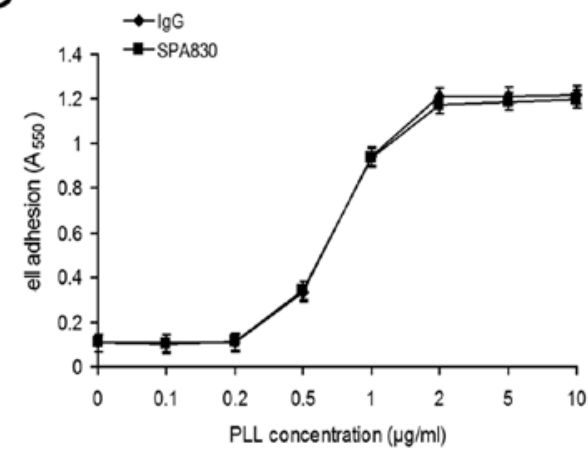

E

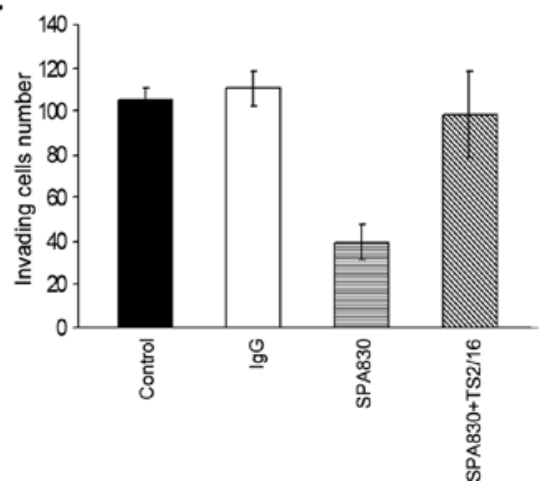

B

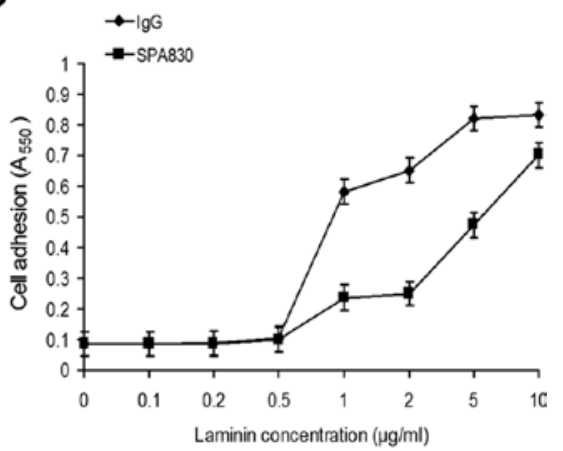

D

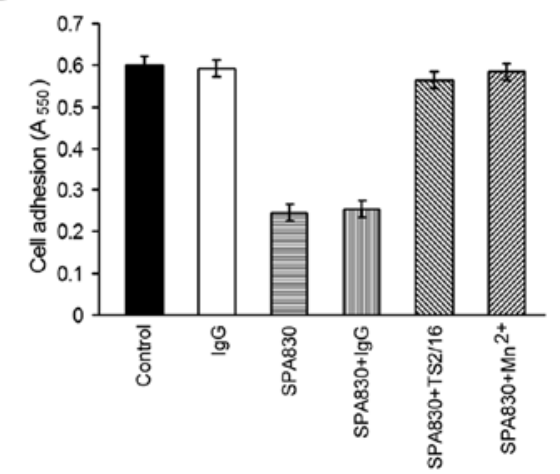

Figure 3. Inhibition of cell surface Hsp90 suppresses integrin-mediated cell adhesion and invasion. (A-C) A series of 96-well plates were coated with serially diluted fibronectin (FN, A), laminin (B) or poly-L-lysine (PLL; C). Cells were plated in the presence of either the anti-Hsp90 antibody SPA830 (10 $\mu \mathrm{g} / \mathrm{ml})$ or control IgG. Thirty minutes later, adherent cells were stained with crystal violet. (D) After treatment with SPA830 or control IgG for 12 h, cells were plated onto FN-coated wells for $30 \mathrm{~min}$. Integrin $\beta 1$-activating antibody was then added to reach a concentration of $1 \mu \mathrm{g} / \mathrm{ml}$ and the cells were incubated for $20 \mathrm{~min}$. Values represent the mean \pm SD A550 of the extracted dye from wells that were plated in quintuplicate. (E) PC3 cells were treated with SPA830 (10 $\mu \mathrm{g} / \mathrm{ml})$ with or without integrin $\beta 1$-activating antibody $(1 \mu \mathrm{g} / \mathrm{ml})$. Invasion was assessed using the Boyden Chamber assay. Values represent the mean \pm SD of three independent experiments. ${ }^{*} \mathrm{P}<0.05,{ }^{* *} \mathrm{P}<0.001$. 
A

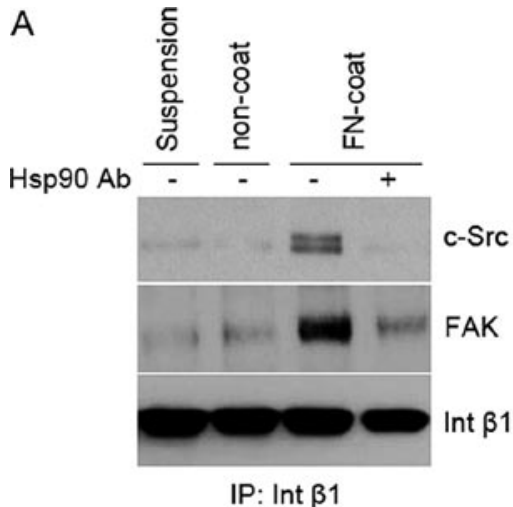

$\mathrm{B}$

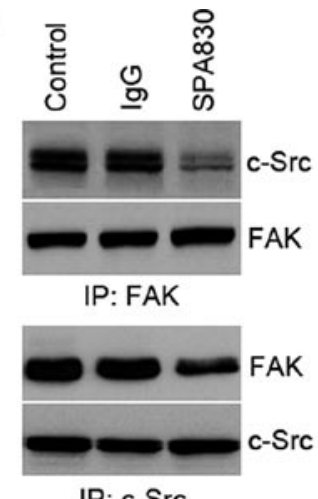

C

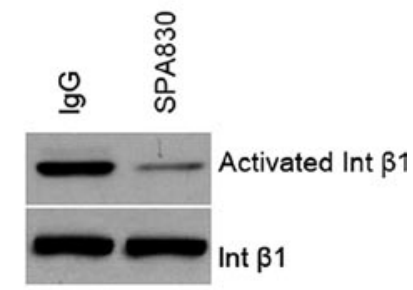

Figure 4. Inhibition of cell surface Hsp90 disrupts the FN-induced association of integrin $\beta 1$, c-Src, and FAK. PC3 cells were maintained in suspension, replated in the absence of $\mathrm{FN}$, or replated in the presence of $\mathrm{FN}$ with or without anti-Hsp90 antibody SPA830 (10 $\mu \mathrm{g} / \mathrm{ml})$ treatment. After cell lysis, integrin $\beta 1$ proteins were immunoprecipitated and examined for the presence of associated c-Src or FAK (A). The association between FAK/c-Src and integrin $\beta 1$ in the active conformation were also determined by IP (B and C).

permeabilization procedure keeps the cells intact and only allows immunostaining on the cell surface. We found that Hsp90 was localized on the resting PC3 cell membrane (Fig. 1A). However, in the $0.1 \%$ Triton X-100-permeabilized cells, Hsp90 immunostaining was observed mainly in the cytoplasm and also in the nucleus (Fig. 1A). Furthermore, cell surface Hsp90 was significantly expressed at the leading edge of migrating cells (Fig. 1B) in a wound healing assay, indicating its essential role in cell motility.

In addition, a biotinylation assay of surface proteins was carried out to confirm the presence of cell surface Hsp90 on PC3. Cell surface proteins were biotinylated and then precipitated by binding to streptavidin agarose beads. Intracellular Hsp90 did not bind to the streptavidin agarose beads and thus was not identified on Western blot analysis (Fig. 1C).

The anti-Hsp90 antibody SPA830 is not internalized. The commercial anti-Hsp90 antibody SPA830 specifically recognizes the C-terminal domain of Hsp90. After treating PC3 cells with fluorochrome-labeled SPA830, we did not observe internalization even after incubation for $8 \mathrm{~h}$ (Fig. 2). Therefore, this antibody can be used to specifically target cell surface Hsp90.

Inhibition of cell surface Hsp90 suppresses integrin-mediated adhesion and invasion. The ability of cell surface Hsp90 to modulate PC3 cell adhesion to the ECM was evaluated. Compared with control cells, PC3 cells treated with the antiHsp90 antibody SPA830 displayed significantly decreased ECM adhesion capacity (Fig. 3A and B). Adhesion to poly-Llysine, which does not engage integrins, was not affected by cell surface Hsp90 inhibition (Fig. 3C). These results indicate that integrin affinity for ECM ligands was down-regulated by the inhibition of cell surface Hsp90.

Conformational changes have been suggested as a possible structural basis that may explain changes in integrin affinity and function (13). If cell surface Hsp90 inhibition did indeed inhibit cell adhesion by causing integrins to convert to an inactive conformation, then treatment of cells with integrin- activating antibody should be able to reverse the inhibitory effects. Our result showed that the integrin $\beta 1$-activating antibody (TS2/16 mAb, $1 \mu \mathrm{g} / \mathrm{ml}$, Thermo Scientific, Rockford, IL) significantly reversed the inhibitory effects of SPA830, while a control IgG antibody did not exert this effect (Fig. 3D).

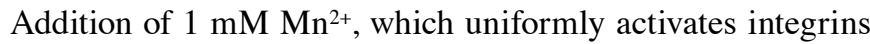
by inducing an active conformation (21), restored cell adhesion (Fig. 3D). Therefore, the inhibition of cell surface Hsp90 inactivated integrins by converting them into an inactive conformation.

Cell migration is an initial step in cell invasion. By using a Boyden chamber assay, we observed that the anti-Hsp90 antibody SPA830 significantly impeded PC3 cells migration (it was inhibited by 63\%), while treatment with a control IgG antibody was unable to induce this effect. The integrin $\beta 1$-activating antibody reversed the inhibitory effects of SPA830, indicating that it inhibited integrin $\beta 1$-mediated cell migration (Fig. 3E).

Inhibition of cell surface Hsp90 disrupts the FN-induced association of integrin $\beta 1$ with FAK and $c$-Src. The ECM-dependent dynamic assembly of focal adhesion molecules, including the $\mathrm{FN}$-induced association of integrin $\beta 1$ with c-Src or FAK, plays a key role in cell migration (13-15). In many signaling contexts, this complex acts to control the turnover of focal cell adhesions during cell migration. We examined whether the cell-impermeable Hsp90 antibody interfered with the ECM-stimulated interaction between FAK/c-Src and integrin $\beta 1$. After PC3 cells were treated with serum-free media containing SPA830 or control IgG for $16 \mathrm{~h}$, the cells were replated on culture dishes coated with FN and allowed to incubate for $1 \mathrm{~h}$. While cells that were cultured in suspension or cells that had been plated on uncoated culture dishes for $1 \mathrm{~h}$ failed to demonstrate an association between FAK/c-Src and integrin $\beta 1$, significant formation of this complex was observed with the cells cultured on FN-coated dishes. However, these complexes failed to form in cells treated with SPA830 (Fig. 4A and B). In addition, we found that the proportion of activated integrin $\beta 1$ was significantly 
A

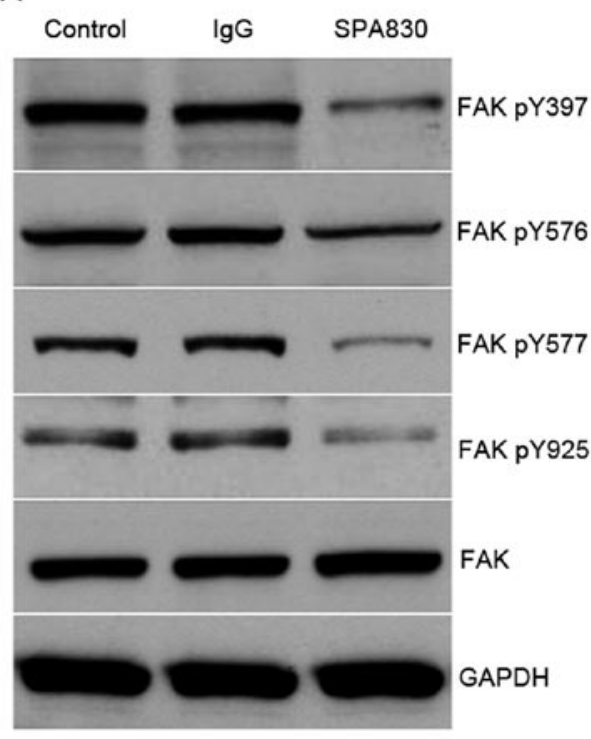

B

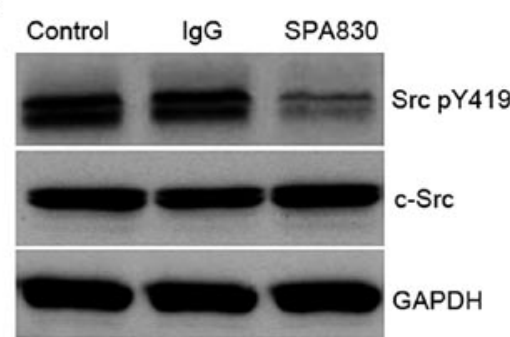

C

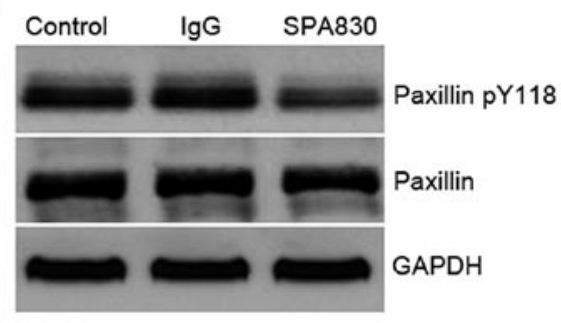

D

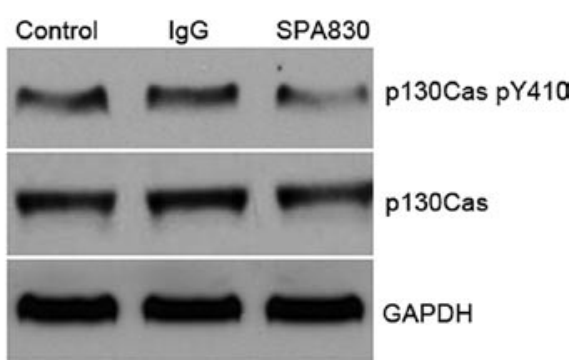

Figure 5. Inhibition of cell surface Hsp90 leads to FAK/c-Src inactivation. PC3 cells were treated with serum-free media containing SPA830 (10 $\mu$ g/ml) or control IgG for $16 \mathrm{~h}$, and replated onto FN-coated wells. After incubation for $1 \mathrm{~h}$, the cells were lysed. The expression of FAK that was phosphorylated at its Y397, Y576, Y577, Y925 residues (A) as well as phosphorylated c-Src (pY419, B), paxillin (Y118, C), and p130Cas (Y410, D) were evaluated by Western blot analysis.

decreased by treatment with SPA830 (Fig. 4C). Along with the available data regarding adhesion and cell migration, our study suggests that integrin $\beta 1$ plays an important role in cell surface Hsp90 signaling during tumor metastasis.

FAK/c-Src is rapidly inactivated after cell surface Hsp90 inhibition. The signals initiated by ECM-integrin interactions are transduced into cells through multiple intracellular signaling pathways, including a cascade of tyrosine phosphorylation events. Two important mediators of this process are focal adhesion kinase (FAK) (14) and Src (15), two non-receptor tyrosine kinases. Clustering of the integrins facilitates the autophosphorylation of tyrosine 397, which increases the catalytic activity of FAK. The motif surrounding tyrosine 397 facilitates the binding of SH2 (Src homology 2) domain containing proteins whose most important member is Src $(13,14)$. The catalytic loop in FAK harbors two tyrosine residues, Y576 and Y577, whose phosphorylation is induced by the binding of Src to FAK-tyrosine 397 and is necessary for FAK to achieve its full kinase activity $(13,14)$. The C-terminal domain of FAK mediates interactions with proteins possessing SH3 domains, such as p130CAS. Furthermore, it contains an important tyrosine residue, Y925. After this residue is phosphorylated, it interacts with several adaptor proteins that localize FAK to integrins via scaffolding proteins (such as paxillin) $(13,14)$. Phosphorylated FAK or c-Src exhibits a higher kinase activity than the unphosphorylated form of the protein, and correlates with more aggressive phenotypes $(14,15)$.

Therefore, we sought to determine whether the effects that cell surface Hsp90 inhibition has on motility are mediated through FAK/c-Src signaling. After PC3 cells were treated with serum-free media containing SPA830 or control IgG for $16 \mathrm{~h}$, the cells were replated on culture dishes coated with FN and allowed to incubate for $1 \mathrm{~h}$. FAK phosphorylation was reduced at tyrosine 397, tyrosine 576, tyrosine 577, and tyrosine 925 in SPA830-treated cells $(15 \mu \mathrm{g} / \mathrm{ml})$ compared to control cells (Fig. 5A). Of note, total FAK protein levels were not significantly changed. The autophosphorylation of c-Src at tyrosine 419 (Y419) is a surrogate marker of its activity. SPA830 treatment led to a dramatic reduction in c-Src autophosphorylation (Fig. 5B). Additionally, total c-Src protein levels were not significantly changed. We also examined the activity of two downstream signaling molecules. Tyrosine phosphorylation of paxillin (Y118) and p130Cas (Y410) were significantly reduced, respectively, upon inhibition of cell surface Hsp90 (Fig. 5C and D). These findings suggest that the inhibition of cell surface Hsp90-induced invasion of PC3 cells may be a result of FAK/c-Src inactivation.

The FAK/c-Src pathway is required for cell surface Hsp90 to exert its effect on the invasiveness of PC3 cells. To substan- 


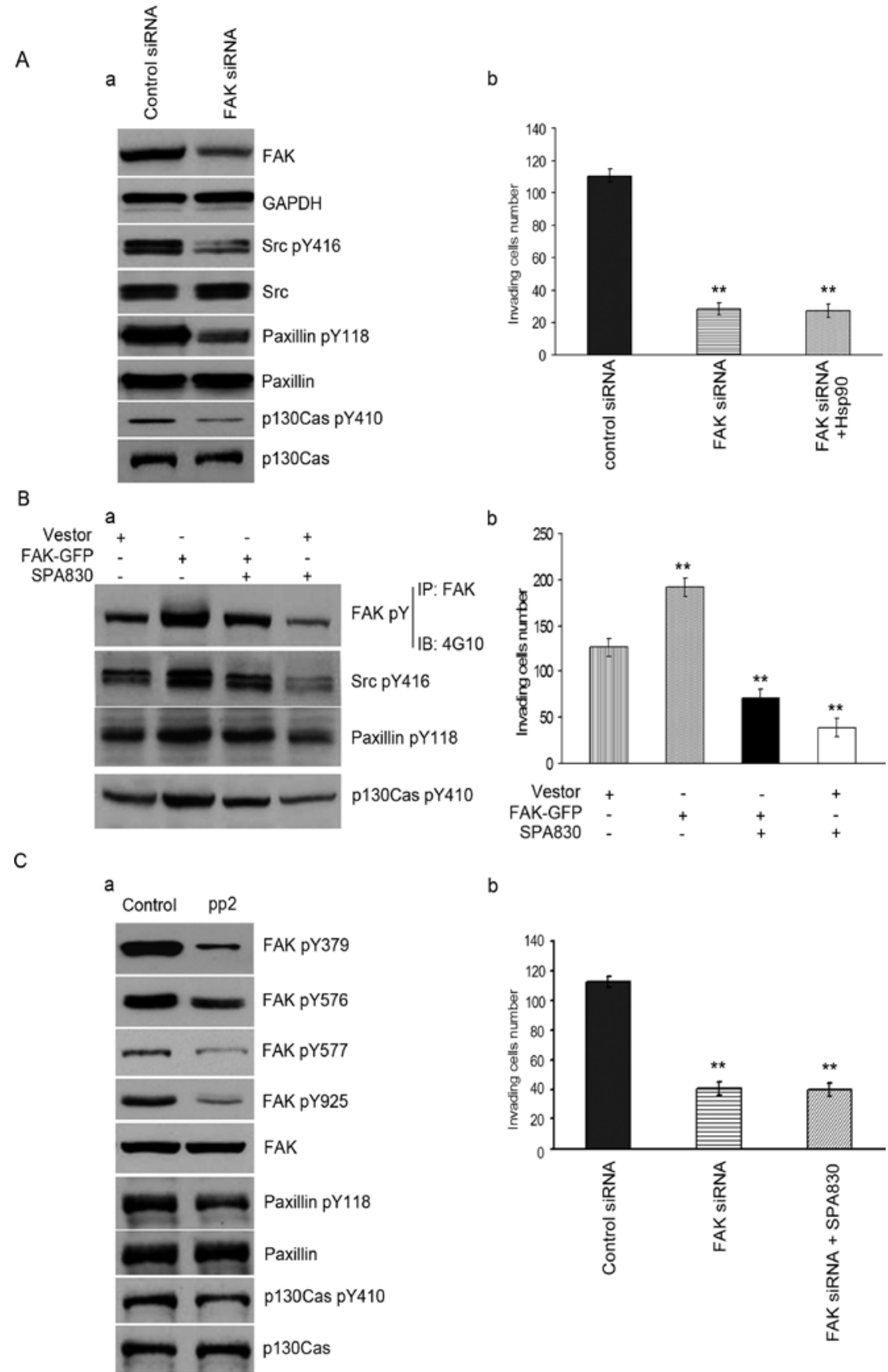

Figure 6. FAK/c-Src pathway is essential for cell surface Hsp90 to exert its effect on PC3 cell invasion capacity. (A) FAK plays an important role in invasion of PC 3 cells mediated by cell surface Hsp90. (a) Cells that had been transfected with FAK siRNA or control siRNA for $36 \mathrm{~h}$ were detached and replated onto FN-coated wells. After incubation for $1 \mathrm{~h}$, the cells were lysed. Verification of FAK knockdown, as well as the phosphorylation of c-Src, paxillin and p130Cas, was determined by Western blot analysis. (b) Cells transfected with FAK siRNA or control siRNA for $36 \mathrm{~h}$ were treated with SPA830 (10 $\mu \mathrm{g} / \mathrm{ml})$ or control $\mathrm{IgG}$ for $8 \mathrm{~h}$ and then were harvested for invasion assay. The results were presented as mean \pm SD. (B) Inhibition of cell surface Hsp90 reversed invasion of PC 3 cells stimulated by overexpression of FAK. (a) PC3 cells were transfected with FAK-GFP plasmid or empty vector. In $36 \mathrm{~h}$, the transfected cells were treated with serum-free media containing SPA830 $(10 \mu \mathrm{g} / \mathrm{ml})$ for $16 \mathrm{~h}$ and then were replated onto FN-coated wells. After incubation for $1 \mathrm{~h}$, the cells were lysed. Immunoprecipitated (IP) FAK was blotted for phosphotyrosine (pY). Total cell lysates were probed for the phosphorylated c-Src, paxillin and p130Cas. (b) PC3 cells that have been transfected with FAK plasmid for $36 \mathrm{~h}$ were harvested for invasion assay as described above. (C) c-Src is involved in invasion of PC3 cells mediated by cell surface Hsp90. (a) PC3 cells pretreated with PP2 $(20 \mu \mathrm{M})$ or DMSO for $48 \mathrm{~h}$ were replated onto FN-coated wells. After incubation for $1 \mathrm{~h}$, cells were lysed and analyzed by Western blot analysis. (b) PC3 cells pretreated with PP2 or DMSO for $48 \mathrm{~h}$ were treated with serum-free media containing SPA830 or control IgG for $8 \mathrm{~h}$. An invasion assay was carried out as described above.

tiate this result, we knocked down FAK expression by treating PC3 cells with FAK siRNA or overexpressed FAK expression by FAK-GFP transfection, and then examined the effects on the invasion capacity of PC 3 cells. Cells that have been transfected with FAK-GFP for $36 \mathrm{~h}$ were treated with serum-free media containing SPA830 or control IgG for $16 \mathrm{~h}$. The cells were replated on culture dishes coated with FN and allowed to incubate for $1 \mathrm{~h}$. We found that the FAK knockdown caused decreased tyrosine phosphorylation of c-Src, paxillin, and p130Cas (Fig. 6Aa). There was a dramatic inhibition of 
invasion in FAK-depleted PC3 cells (Fig. 6Ab). In addition, we determined whether the inhibition of cell surface Hsp90 could reverse the cell invasion stimulated by overexpression of FAK. Transfection of FAK-GFP plasmid restored phosphorylated FAK, c-Src, paxllin and p130Cas protein levels inhibited by the Hsp90 antibody SPA830 (Fig. 6Ba). Overexpression of FAK reversed the cell invasion inhibited by SPA830 (Fig. 6Bb). These results suggest that FAK activation may play a critical role in these processes.

To further characterize the interaction between FAK and c-Src, we examined the effect of Src inhibition on FAK phosphorylation. Our results showed that upon treatment of PC3 cells with Src inhibitor PP2, phosphorylation of FAK at its Y397, Y576, Y577 and Y925 residues was significantly reduced (Fig. 6Ca). Phosphorylation of paxillin and p130Cas was also reduced in PP2-treated cells (Fig. 6Ca). Compared with control cells, the invasive capacity of PC 3 cells was also decreased in the presence of PP2 (Fig. 6Cb). However, in the presence of SPA830, neither FAK knockdown (Fig. 6Ab) nor PP2 treatment (Fig. 6Cb) further retarded invasion. Taken together, these data suggest that the inhibition of cell surface Hsp90 decreases the invasiveness of PC 3 prostate cancer cells through the FAK/Src pathway.

\section{Discussion}

The blockade of key molecules in the signaling pathways that regulate tumor cell invasion is one of the most promising potential treatment strategies in modern cancer therapy (2). Cell surface Hsp90 has been recently found to be implicated in cell migration and may be a promising target for cancer therapy $(4,22)$. Prostate cancer begins as an androgen-dependent disease and frequently progresses to a castrate-resistant state in its late stages. PC3 cells are most commonly used cellular prototype of advanced prostate cancer and they exhibit an intrinsic migratory ability. Using an immunofluorescent live cell staining technique and surface biotinylation assay, we showed that Hsp90 was expressed on the surface of PC3 cells. Notably, cell surface Hsp90 was found to be localized at the leading edge of migrating PC3 cells in a wound-healing assay, suggesting that it may play a critical role in cell motility. In our study, inhibiting cell surface Hsp90 using a cell-impermeable antibody decreased prostate cancer cell adhesion and invasion. Dynamic focal adhesion formation is critical for cell migration and invasion. The anti-Hsp90 antibody SPA830 not only decreased the proportion of integrin $\beta 1$ that was in an activated conformation, but it also inactivated FAK and c-Src, two major regulators of focal adhesion turnover, as well as their downstream molecules. Taken together, these findings provide mechanistic insight into the role of cell surface Hsp90 in prostate cancer cell motility and invasiveness.

The invasiveness of malignant cells depends on their ability to adhere to and invade the surrounding stroma. Integrins are a family of cell surface adhesion receptors that heterodimerize to produce more than 20 different heterodimers capable of mediating a wide range of cellular responses (13). Changes in integrin structure and/or expression are frequently associated with malignant transformation and tumor progression $(13,23)$. Each integrin binds to specific ECM components and clusters in the membrane to form focal cell-ECM adhesions. There are extensive connections between the integrins and the downstream molecules FAK, and Src family kinases at the heart of these adhesion interactions as well as cell migration signaling $(23,24)$. Reducing integrin $\beta 1$ expression by interfering with FAK/Src signaling (25), treating cells with an anti-integrin $\beta 1$ antibody (26), or by administering high dose radiation treatment (19) significantly decreased the adhesion, motility and invasion capacity of PC 3 cells. Additionally, integrin $\beta 1$ is required for bombesin-dependent pro-MMP-9 activation (17). Tsutsumi et al found that inhibiting cell surface Hsp90 interfered with the FN-stimulated interaction between c-Src and integrin $\beta 1$ (6). We showed that the associations between integrin $\beta 1$, FAK, and c-Src were disrupted when PC3 cells were treated with anti-Hsp90 antibodies. In addition, treatment with an anti-Hsp90 antibody decreased the proportion of integrin $\beta 1$ that was in an active conformation. Activating integrin $\beta 1$ using a specific antibody following anti-Hsp90 antibody treatment significantly restored the adhesion and invasive capacity of PC 3 cells. These findings indicate that integrin $\beta 1$ may be a client protein of cell surface Hsp90.

Although more than 100 intracellular Hsp90 client proteins have been identified, much less is known about cell surface Hsp90 client proteins $(4,22)$. Recently, cell surface Hsp90 was reported to interact with extracellular domain of ErbB2, a protein whose intracellular kinase domain is already known to interact with Hsp90 (10). In addition, several components of the Hsp90 chaperone complex, including Hsp70, Hop, and P23, have been shown to be present extracellularly (27). Hsp90 was also detected in conditioned media of HT-1080 fibrosarcoma cells and it has been reported to bind to and activate MMP-2 (9). We also observed the expressions of Hsp70 and Hop on cell surface of PC3 cells (data not shown). These data suggest that cell surface Hsp90, much like its intracellular counterpart, is a multifunctional molecule that interacts with several proteins. Although cell surface Hsp90 did not co-patch with integrin $\beta 1$ upon crosslinking (data not shown), we speculate that cell surface Hsp90 may modulate integrin function directly or indirectly, although these findings need to be validated in future studies.

Integrins are functionally and physically interconnected through FAK, which is recruited and phosphorylated at sites of focal adhesions upon integrin aggregation and serves as a bridge between growth factor receptors and integrin cytoplasmic tails (13). Its downstream target, c-Src, as well as paxillin and p130Cas (two scaffold proteins involved in integrin signaling), have been proposed to be required for integrin-mediated migration and invasion (13). Our study clearly demonstrated that the FAK/c-Src complex is required in order for PC 3 cells to possess invasive capacity and that the inhibition of cell surface Hsp90 suppresses both FAK/Src activity and the invasion capacity of PC3 cells. Inhibition of cell surface Hsp90 can reverse the cell invasion stimulated by overexpression of FAK. Interestingly, neither FAK knockdown by siRNA treatment nor the inhibition of Src by PP2 treatment in the presence of Hsp90 antibody further suppressed invasion. These data indicate that the activation of the FAK/c-Src signaling pathway is the key downstream event in cell surface Hsp90 signaling that regulates the invasion capacity of PC 3 cells. 
In conclusion, our study has identified a new signaling pathway that originates with the inhibition of cell surface Hsp90 and leads to the suppression of integrin function and FAK/c-Src inactivation. In this study, administration of an antibody that was targeted against cell surface Hsp90 was very effective at blocking tumor cell adhesion and migration. This suggests that the inhibition cell surface Hsp90 could be explored as a potential novel molecular therapeutic approach to inhibit cancer invasion in primary prostate cancers as well as other malignancies.

\section{Acknowledgements}

This study was supported by the Scientific Research Foundation for the Returned Overseas Chinese Scholars from the Ministry of Education, China. We are grateful to Dr J.T Parsons for his provision of GFP-FAK plasmid and to Dr Jennifer S. Isaacs (Medical University of South Carolina, USA) for her kindly advice in the immunofluorescence and biotinylation assay.

\section{References}

1. Jemal A, Siegel R, Ward E, Hao Y, Xu J, Murray T and Thun MJ: Cancer statistics, 2008. CA Cancer J Clin 58: 71-96, 2008.

2. Geiger TR and Peeper DS: Metastasis mechanisms. Biochim Biophys Acta 1796: 293-308, 2009.

3. Tsutsumi S, Beebe K and Neckers L: Impact of heat-shock protein 90 on cancer metastasis. Future Oncol 5: 679-688, 2009.

4. Tsutsumi S and Neckers L: Extracellular heat shock protein 90: a role for a molecular chaperone in cell motility and cancer metastasis. Cancer Sci 98: 1536-1539, 2007.

5. Xu W and Neckers L: Targeting the molecular chaperone heat shock protein 90 provides a multifaceted effect on diverse cell signaling pathways of cancer cells. Clin Cancer Res 13 $1625-1629,2007$.

6. Tsutsumi S, Scroggins B, Koga F, et al: A small molecule cellimpermeant Hsp90 antagonist inhibits tumor cell motility and invasion. Oncogene 27: 2478-2487, 2008.

7. Stellas D, Karameris A and Patsavoudi E: Monoclonal antibody 4C5 immunostains human melanomas and inhibits melanoma cell invasion and metastasis. Clin Cancer Res 13: 1831-1838, 2007.

8. Becker B, Multhoff G, Farkas B, Wild PJ, Landthaler M, Stolz W and Vogt T: Induction of Hsp90 protein expression in malignant melanomas and melanoma metastases. Exp Dermatol 13: 27-32, 2004

9. Eustace BK, Sakurai T, Stewart JK, et al: Functional proteomic screens reveal an essential extracellular role for hsp90 alpha in cancer cell invasiveness. Nat Cell Biol 6: 507-514, 2004.
10. Sidera K, Gaitanou M, Stellas D, Matsas R and Patsavoudi E: A critical role for HSP90 in cancer cell invasion involves interaction with the extracellular domain of HER-2. J Biol Chem 283: 2031-2041, 2008

11. Cid C, Regidor I, Poveda PD and Alcazar A: Expression of heat shock protein 90 at the cell surface in human neuroblastoma cells. Cell Stress Chaperones 14: 321-327, 2009.

12. Sidera K, Samiotaki M, Yfanti E, Panayotou G and Patsavoudi E: Involvement of cell surface HSP90 in cell migration reveals a novel role in the developing nervous system. J Biol Chem 279: 45379-45388, 2004.

13. Goel HL, Li J, Kogan S and Languino LR: Integrins in prostate cancer progression. Endocr Relat Cancer 15: 657-664, 2008.

14. Zhao J and Guan JL: Signal transduction by focal adhesion kinase in cancer. Cancer Metastasis Rev 28: 35-49, 2009.

15. Araujo $J$ and Logothetis C: Targeting Src signaling in metastatic bone disease. Int J Cancer 124: 1-6, 2009.

16. Edick MJ, Tesfay L, Lamb LE, Knudsen BS and Miranti CK: Inhibition of integrin-mediated crosstalk with epidermal growth factor receptor/Erk or Src signaling pathways in autophagic prostate epithelial cells induces caspase-independent death. Mol Biol Cell 18: 2481-2490, 2007.

17. Festuccia C, Angelucci A, Gravina G, Eleuterio E, Vicentini C and Bologna M: Bombesin-dependent pro-MMP-9 activation in prostatic cancer cells requires beta1 integrin engagement. Exp Cell Res 280: 1-11, 2002.

18. Franzen CA, Amargo E, Todorovic V, et al: The chemopreventive bioflavonoid apigenin inhibits prostate cancer cell motility through the focal adhesion kinase/Src signaling mechanism. Cancer Prev Res (Phila) 2: 830-841, 2009.

19. Simon EL, Goel HL, Teider N, Wang T, Languino LR and Fitzgerald TJ: High dose fractionated ionizing radiation inhibits prostate cancer cell adhesion and beta(1) integrin expression. Prostate 64: 83-91, 2005.

20. Sridhar SC and Miranti CK: Tetraspanin KAI1/CD82 suppresses invasion by inhibiting integrin-dependent crosstalk with c-Met receptor and Src kinases. Oncogene 25: 2367-2378, 2006.

21. Miao H, Burnett E, Kinch M, Simon E and Wang B: Activation of EphA2 kinase suppresses integrin function and causes focaladhesion-kinase dephosphorylation. Nat Cell Biol 2: 62-69, 2000.

22. Sidera K and Patsavoudi E: Extracellular HSP90: conquering the cell surface. Cell Cycle 7: 1564-1568, 2008.

23. Huveneers S and Danen EH: Adhesion signaling-crosstalk between integrins, Src and Rho. J Cell Sci 122: 1059-1069, 2009.

24. Huveneers S, Arslan S, van de Water B, Sonnenberg A and Danen EH: Integrins uncouple Src-induced morphological and oncogenic transformation. J Biol Chem 283: 13243-13251, 2008.

25. Zhang J, Park SI, Artime MC, et al: AFAP-110 is overexpressed in prostate cancer and contributes to tumorigenic growth by regulating focal contacts. J Clin Invest 117: 2962-2973, 2007.

26. Scott LJ, Clarke NW, George NJ, Shanks JH, Testa NG and Lang SH: Interactions of human prostatic epithelial cells with bone marrow endothelium: binding and invasion. Br J Cancer 84: 1417-1423, 2001.

27. Eustace BK and Jay DG: Extracellular roles for the molecular chaperone, hsp90. Cell Cycle 3: 1098-1100, 2004. 